\section{P40 IS ROUTINE LIVER FUNCTION TESTING NECESSARY FOR PATIENTS RECEIVING LATENT TUBERCULOSIS TREATMENT?}

S Christie, J Potter. North Middlesex University Hospital, London, UK

\subsection{6/thorax-2021-BTSabstracts. 150}

Introduction Tuberculosis was the leading cause of mortality from infectious disease until the covid-19 pandemic. Over a quarter of the world's population is estimated to have latent tuberculosis infection (LTBI), with 5-10\% expected to develop active TB. Hepatotoxicity is a well described side-effect of anti-tuberculosis medication, often leading to disruptions in treatment. Currently, NICE guidelines recommend careful liver function test (LFT) monitoring of patients on anti-tuberculosis medication with liver disease, abnormal baseline LFTs or with history of alcohol or hepatotoxic drug misuse. However, there is no specific guidance on LFT monitoring for routine treatment.

Methods All patients with LTBI in a single centre were identified from hospital records over a 3-year period (January 2018-December 2020). Clinical notes and blood test results were analysed for all patients, identifying any drug induced liver injury (DILI). Frequency and timing of LFT monitoring once starting treatment was reviewed and correlated with patient symptoms.

Results Over a 3-year period, 180 patients received treatment for LTBI. 169 patients (94\%) had baseline LFTs measured. 7 patients $(3.9 \%)$ had deranged LFTs during their treatment, with $3(1.7 \%)$ meeting the criteria for DILI. 2 of the 3 patients (66\%) with DILI were symptomatic prompting blood tests, 1 patient (33\%) had abnormal baseline LFTs. Of the 7 patients with LFT derangement during treatment, 4 (57\%) had deranged baseline LFTs, 4 (57\%) were symptomatic. Only 1 patient had neither symptoms nor abnormal baseline LFTs, but had abnormal baseline renal function requiring regular monitoring. Conclusions These results suggest that risk of DILI is present but relatively low in patients receiving treatment for LTBI. Most patients from this cohort with DILI were either high risk as per NICE guidance or symptomatic prompting LFT checking. Therefore, routine monitoring of LFTs for all patients on LTBI treatment is unlikely to be cost-effective or change treatment outcomes. Instead, LFT monitoring should be considered specifically for patients that are high risk or with symptoms of DILI.

\section{REFERENCES}

1. National Institute for Health and Care Excellence (NICE) (2016) Tuberculosis. Available at https://www.nice.org.uk/guidance/ng33

2. Global tuberculosis report 2020. Geneva: World Health Organization; 2020. Licence: CC BY-NC-SA 3.0 IGO.

\section{P41 PSEUDOMONAS AERUGINOSA: BURDEN, TREATMENT AND OUTCOMES IN A LONG TERM VENTILATION SERVICE}

HJ Carlin, RE Sobala, TB Fretwell, S Shakir, K Cattermole, P McCallion, A Royston, J Davison, J Lumb, H Tedd, B Messer, A De Soyza. Newcastle Upon Tyne Hospitals NHS Foundation, Newcastle upon Tyne, UK

\subsection{6/thorax-2021-BTSabstracts.151}

Introduction Pseudomonas aeruginosa (PA) is a significant respiratory pathogen but is not well described in patients requiring long term ventilation (LTV). Whilst national guidance exists for the management of PA in the bronchiectasis population, standards for sputum surveillance and the use of nebulised antibiotics (NA) or long term macrolides (LTM) in the LTV population have not been established.

Our aim was to record the rate of PA and describe its treatment, in a large cohort of LTV patients.

Methods Retrospective cohort study using the regional LTV service database to identify patients. Electronic patient records were analysed for LTV indication and treatment with NA and LTM, and cross-referenced with regional microbiology datasets: specifically, positive results for Pseudomonas, Serratia, Proteus and/or Burkholderia spp.

Results We identified 837 patients under the regional LTV service. 52/837 (6.2\%) were ventilated via tracheostomy. 57/ $837(6.8 \%)$ used a cough assist device alone and the remainder used non-invasive ventilation.

Figure 1 shows prevalence of PA by diagnostic subgroup; the burden of PA in bronchiectasis and CF was high as expected, however there was also a high burden of disease in patients with spinal cord injuries and primary muscle disorders, such as Duchenne Muscular Dystrophy.

Of the PA positive cohort, 45/146 (30.8\%) were treated with NA: colistin (64.4\%), an alternative NA (11.1\%) or a combination (24.4\%). 49.2\% were taking long term macrolides: alone $(56.2 \%)$ or combined with NA (43.7\%).

Commencement of NA was poorly documented, hindering assessment of PA suppression or effects on emerging pathogens. Proteus/Serratia/Burkholderia spp. were isolated in 5.1\% of the LTV cohort, of which $38 \%$ were co-colonising patients with PA.

Conclusions PA is common and seen across a diverse number of LTV indications, including neuromuscular conditions not traditionally associated with high bacterial load. PA prevalence was higher in tracheostomy patients. Many patients are treated with NA but the recording of eradication rates and sputum surveillance is low. The rate of 3 sentinel emergent pathogens was low but this may reflect the low rate of sputum

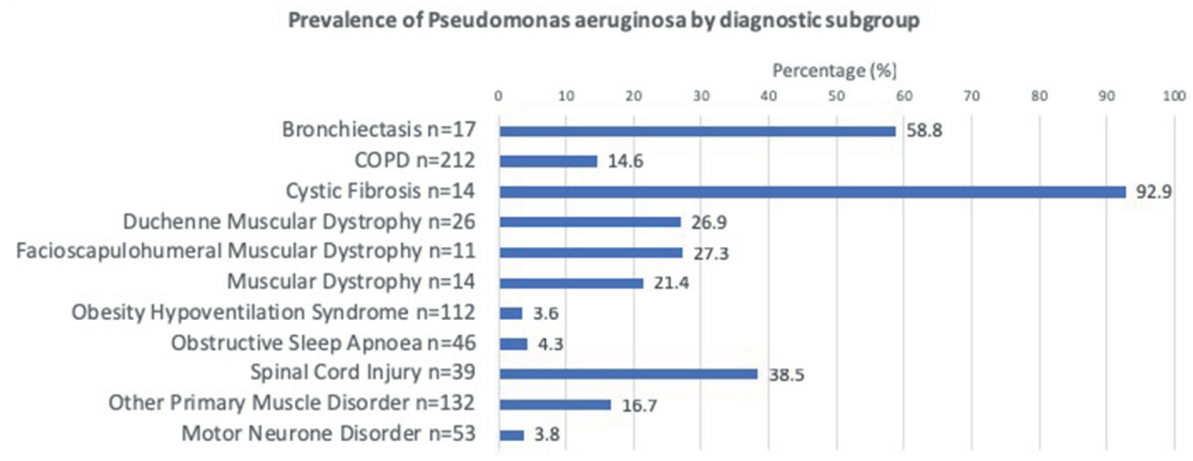

Abstract P41 Figure 1 Prevalence of pseudomonas aeruginosa by diagnostic subgroup 
surveillance. This study highlights a need for further studies and development of guidance on pseudomonas in patients on LTV.

\section{Diagnostics and monitoring of asthma and co-morbidities}

\section{P42 ASTHMA TREATMENT ADHERENCE CHECKS: PRESENT AND FUTURE}

N Stewart-Kelcher, A Patel, S Rahman, A Hearn, C Roxas, L Green, L Thomson, M Fernandes, AM Nanzer, DJ Jackson, J Dhariwal, G d'Ancona. Guy's and St Thomas' NHS Foundation Trust, London, UK

\subsection{6/thorax-2021-BTSabstracts. 152}

Introduction In spite of its importance in optimising clinical outcomes and determining whether an escalation in therapy is indicated (e.g. initiating a biologic agent), the difficulty in identifying suboptimal adherence to asthma therapy persists. The most common method used to measure adherence by pharmacists in hospital asthma centres is the prescription refill check (PRC), but it is both flawed (e.g. assumption made that therapy collected has been appropriately administered) and can be time consuming to collect and interpret. This may therefore make it a barrier to effective and timely asthma management. The aim of this retrospective study was to quantify the time taken to complete a PRC from primary care using data retrieved from shared local care records (LCR) versus those obtained by contacting general practice (GP) directly, and the additional time taken when there was a need to obtain hospital prescription data.

Methods Data were scrutinised for patients for whom a PRC was conducted between August 2019 and May 2021 to ascertain the time interval taken between identification of the need for the PRC and its availability on the patient's electronic record.

Results Data for 885 patients were scrutinised and are illustrated in table 1.

A PRC using direct data extraction from a LCR took on average less than 2 days to complete. In contrast, there was an eleven fold increase in the time taken to complete a PRC when GP's had to be contacted. Retrieval of data from another hospital also added a delay to availability of prescription data.

Conclusions Given the frequency of sub-optimal adherence and its impact, access to robust and complete data needs to be efficient. The stark disparity amongst sources suggests

\begin{tabular}{lll}
$\begin{array}{l}\text { Abstract P42 Table } \\
\text { data by data source }\end{array}$ & Time interval for availability of prescription \\
\hline $\begin{array}{l}\text { Source (number of } \\
\text { patients) }\end{array}$ & $\begin{array}{l}\text { Mean interval in days } \\
\text { between PRC request and } \\
\text { data availability (Std Dev) }\end{array}$ & Range (days) \\
\hline GP $(n=445)$ & $14.7(21.1)$ & $0-182$ \\
GP + Hospital $(n=37)$ & $14.0(12.2)$ & $0-64$ \\
LCR (n=340) & $1.3(3.2)$ & $0-44$ \\
LCR + Hospital $(n=63)$ & $6.1(5.6)$ & $0-34$ \\
\hline
\end{tabular}

that while the utility of electronic monitoring of therapy is established, appropriate direct access to prescription data is essential. This would then streamline the process by reducing demand on healthcare professionals to provide the data, would minimise delays in treatment escalation decisions, and the significant decrease in hospital pharmacy resource needed to obtain this data would allow pharmacist's time to be better spent improving non-adherence rather than detecting it.

\section{P43 EXPERIENCES OF ASTHMA IN THE UK-RESIDENT ADULT SOUTH ASIAN POPULATION: A QUALITATIVE STUDY}

${ }^{1,2} \mathrm{ZK}$ Yusuf, ${ }^{3} \mathrm{D}$ Wensley, ${ }^{4} \mathrm{H}$ Owton, ${ }^{1,2} \mathrm{SJ}$ Singh, ${ }^{5} \mathrm{JAC}$ Allen Collinson. ${ }^{1}$ Department of Respiratory Sciences, University of Leicester, Leicester, UK; ${ }^{2}$ Centre for Exercise and Rehabilitation Science, NIHR Leicester Biomedical Research Centre, University Hospitals of Leicester NHS Trust, Leicester, UK; ${ }^{3}$ Department of Nursing and Midwifery, De Montfort University, Leicester, UK; ${ }^{4}$ School of Education, Childhood, Youth and Sport, Open University, Milton Keynes, UK; ${ }^{5}$ School of Sport and Exercise Science, University of Lincoln, Lincoln, UK

\subsection{6/thorax-2021-BTSabstracts. 153}

Introduction and Objectives South Asian individuals living with asthma in the UK are more likely to experience excess morbidity and increased hospitalisation rates than any other ethnic group. Prevention is an integral part of self- management (Pinnock, 2015). Failure to adhere to prescribed regimens is common amongst this population. This study investigated people's experiences with asthma, including medication adherence, the use of non-pharmacological treatment approaches, and the healthcare professional (HCP)-patient relationship in asthma healthcare.

Methods Using a qualitative approach, fourteen adults (12 female, 2 male, aged between 18-50) who identified as South Asian with a diagnosis of asthma (at least step 2 of the BTS guidelines) took part in semi-structured interviews. Interpretative phenomenological analysis (IPA) was used, informed by a symbolic interactionist (SI) perspective; a micro level theoretical framework which suggests that society is shaped and upheld by social interaction and explores how people make sense of their social world (Carter \& Fuller, 2015).

Results Four themes were developed, focusing on how the asthmatic identity is negotiated, managing medications, seeking non-pharmacological treatments, and the HCP-patient relationship (see figure 1). Despite suffering acute exacerbations, participants questioned whether they identified as asthmatic, which impacted their decision to use preventative medication. Cultural identity was linked to traditional treatments and medication adherence. Characteristics of developing a therapeutic relationship with HCPs were described, including patient involvement and mutual respect. This involved having open discussions on the use non-pharmacological treatments which were linked to participants' cultural identity, illustrating the HCP's desire to be culturally responsive.

Conclusions HCP's should consider an explorative approach to consultation, to develop a culturally aware, therapeutic relationship and consider negotiation in prescribing. This could enhance the patients' ability to self-manage, and reduce resistance to advice and guidance from HCPs. Cultural identity is an important aspect of treatment and should be discussed to 